\title{
Multiscale Multimodal Multicolor Microscopy
}

\author{
Ben N. G. Giepmans* \\ Dept. Cell Biology, UMC Groningen, University of Groningen, Groningen, The Netherlands. \\ * Corresponding author: b.n.g.giepmans@umcg.nl
}

Correlative light microscopy and electron microscopy (CLEM) is a key approach to study structure-function relationships in cell biology. CLEM allows a biological process and building block (molecule, organelle, cell) to be identified and dynamically studied using fluorescent markers followed by high-resolution analysis of the ultrastructural context with EM. In the past decades sample preparation steps, technical approaches, probes, microscopes and image analysis have been optimized to make CLEM a routine approach applied by many labs to date [1]. I will focus on additional modalities for identification of molecules in 'cellular microscopy' currently being explored in our lab.

Although all pre-embedding labelling procedures often lead to extraction of biomolecules, only EM painfully shows the effect on the ultrastructure [3]. Well-established ways to circumvent cellular damage by introducing probes are by using either genetically-encoded tags and/ or small molecules that do not need permeabilization [1] or post-embedding labelling, e.g. Fig. 1. The concession in CLEM for sample preparation not optimal for either modality also counts for the light microscopic analysis: fluorescence is typically poorly retained during EM sample preparation focused on retaining optimal ultrastructure, although probes and procedures are being developed to retain fluorescence during embedding [1]), or fluorescent labels can be targeted post-embedding.

Another challenge in CLEM is to deal with large datasets: CLEM approaches have resulted in a demand to image large regions of interest (ROI), typically easily performed with LM, at the typical resolution for EM (nm-scale). In dynamic vital microscopy followed by EM, this gap will not easily be solved routinely, especially because of the difficulty of correlating data in the axial direction. In fixed samples, matching the scales between the modalities demands large-scale EM [1], which leads to an avalanche of data and the current quest is to identify or fingerprint biomolecules in the nm-range.

Many initiatives try to handle and publish large imaging datasets. Our lab has pioneered placing large-scale EM maps of a variety of cells, tissues and model organisms at full resolution with open access at www.nanotomy.org (Fig.1). Initiatives by others are referred to on this website. Current developments in data sharing, analysis, and interpretation - like automated recognition and machine learning software - will evolve in the next decade and solve data handling challenges. Increasingly open access to high-content multidimensional microscopic data will pave the path for multimodal analysis of the high content datasets. Metadata definition and (online) representation of such multi-dimensional and multi-parameter datasets needs to further develop to meet international consensus.

Cathodoluminescence (CL): In the multimodal microscopes, when electrons hit cathodoluminescent molecules, photons can be collected resulting in localization defined by the electron beam [5]. In addition, new analytical methods are being pioneered which are based on using an electron beam or ion beam to achieve the required lateral localization precision.

Energy dispersive X-ray analysis (EDX): Elemental fingerprinting using EDX is described in a tour-theforce by Somlyo and co-workers 40 years ago [6], who revealed subcellular distribution of elements in muscle using spectroscopic methods. Development in EDX detectors and computer software nowadays allows (semi)routine EDX imaging of $1 \mathrm{kx} 1 \mathrm{k}$ pixel areas to fingerprint biomolecules and probes in the context of ultrastructure (Fig.1) [7]. 
Electron energy loss spectroscopy (EELS): In parallel, elemental analysis is performed using EELS. Leapman and colleagues pioneered EELS imaging in tissue to detect endogenous elements[8]. Similarly, EELS TEM allows detection of particles enriched in certain elements targeted for labelling, such as Quantum dots [9]. Recently, Tsien and co-workers used lanthanides-enriched molecules that can be deposited using specific probes to perform two-color EELS to discriminate targeted molecules and biostructures of interest [10].

CLEM has developed to a semi-routine technique. Major bottlenecks, like retaining fluorescence in EMprepared samples have been overcome. Thus, a combination between nanoscopic fluorescence microscopy and EM is now feasible. CLEM will develop to make the workflow more convenient, faster and more generically available. The data sets will be larger, and protocols for reuse of data will be developed as exemplified by several initiatives to already share large-scale EM data. Nowadays, life science microscopists will increasingly explore the use of the electron beam or ion beam to generate signals that fingerprint or identify biomolecules and structures either directly, or indirectly using to-be-developed probes and bypassing the diffraction limit of light microscopy. This will lead to multidimensional EM with a pleiotropy of signals and molecules detected at nm-scale precision and reveal many current secrets underlying the regulation of life [12].

\section{References:}

[1] de Boer et al., Nat. Methods 6 (2015), p. 503.

[2] Balzarotti et al., Science 6325 (2017), p. 606.

[3] Schnell et al., Nat. Methods 2 (2012), p. 152.

[4] de Beer, Histochem. Cell Biol. 149 (2018), p. 261.

[5] Glenn et al., Sci. Rep. 865 (2012).

[6] Somlyo et al., J. Cell Biol. 3 (1977), p. 828.

[7] Scotuzzi et al., Sci. Rep. (2017), p. 45970.

[8] Leapman et al., Ultramicroscopy 180 (2017).

[9] Nisman et al., J. Histochem. Cytochem. 1 (2004), p. 13.

[10] Adams et al., Cell. Chem. Biol. 11 (2016), p. 1417.

[11] Jiang et al., Curr. Opin. Biotechnol. 130 (2016).

[12] I thank my team members and Jacob Hoogenboom, Delft University of Technology, for discussions.

Our work relevant to this paper is supported by the Netherlands organization for scientific research (ZonMW 91111.006; STW Microscopy Valley 12718; TTW15315).

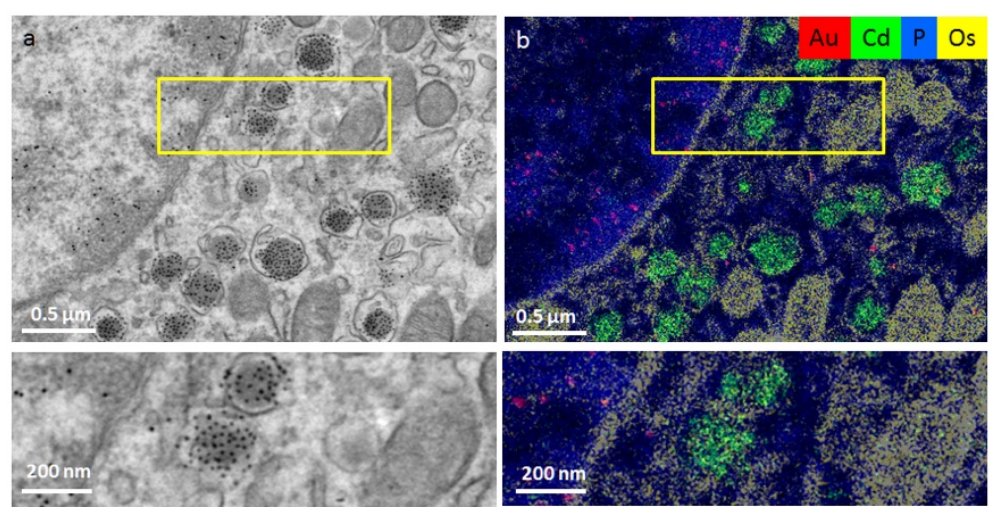

Figure 1. 'ColorEM' using elemental analysis by energy dispersive $\mathrm{X}$-ray imaging. ColorEM: label-free (P), paint (Os) and labeling DNA $(\mathrm{Au})$ and peptides $(\mathrm{Cd})$ is compatible. (a) Part of an islet of Langerhans immuno-labeled for structures in DNA $(10 \mathrm{~nm}$ gold) and insulin (QD). (b) Overlay image of $\mathrm{Au}$ (red), Cd (green), Os (yellow) and P (blue) allows identification of G4 structures (gold labels) and insulin (Cd). Note the localization of Au to heterochromatin enriched in $\mathrm{P}$, whereas the $\mathrm{Cd}$ signal is enclosed within a combination of Os rings and $\mathrm{P}$ that likely identifies phospholipid membranes of the vesicles. Large scale data and full resolution data is available via www.nanotomy.org; Partially reproduced and modified from [7]. 